\title{
PRODUÇÃO ELETROQUÍMICA E UTILIZAÇÃO DE CLORO LIVRE: UMA ABORDAGEM VIA PLANEJAMENTO
}

\author{
R. ANTONELLI ${ }^{1}$, R. F. PIRES ${ }^{1}$, GRANATO A. C. ${ }^{1}$, G. R. P. MALPASS $^{1}$ \\ ${ }^{1}$ Universidade Federal do Triângulo Mineiro, Departamento de Engenharia Química \\ E-mail para contato: raissaantonelli@hotmail.com
}

\begin{abstract}
RESUMO - Este trabalho apresenta a utilização do planejamento de experimentos para quantificar o efeito das variáveis na produção eletroquímica de cloro livre, o qual será utilizado na degradação de poluentes orgânicos juntamente com a luz ultravioleta. A influência das variáveis do sistema (corrente, concentração, espaçamento entre eletrodos e $\mathrm{pH}$ ) foram investigadas, revelando que a corrente é o parâmetro mais significativo, com influencia positiva na resposta. A segunda mais significante é a concentração, também com influencia positiva. Já as variáveis espaçamento e $\mathrm{pH}$ não mostraram muita influencia na produção de cloro. Também, analisaram-se as interações entre as variáveis, sendo que nenhuma interação manifestou significância no processo. Como trabalhos a finalizar, será necessário a execução do planejamento composto central (PCC) para verificação final das condições ótimas de produção. Cogita-se também a execução da degradação de poluentes orgânicos com as condições ótimas do processo para verificar a sua eficácia.
\end{abstract}

\section{INTRODUÇÃO}

O desenvolvimento de um novo produto e/ou processo depende de um grande número de variáveis e o planejamento adequado é indispensável para que resultados confiáveis sejam obtidos e para que análises estatísticas consistentes possam ser realizadas. A metodologia do planejamento experimental, associado à análise de superfícies de respostas, é uma ferramenta fundamentada na teoria estatística, que fornece informações seguras sobre o processo, minimizando o empirismo que envolve técnicas de tentativa e erro (BOX et al., 1978). No entanto, segundo Costa e Almeida (2011), para que o uso dessa metodologia atinja os objetivos desejados, é necessário haver uma integração entre o processo, a estatística e o bom senso, tanto da equipe responsável pela montagem dos experimentos, quanto da equipe responsável pela análise estatística e estratégica dos resultados. Neste trabalho foi desenvolvido um planejamento fatorial $2^{4} \mathrm{com}$ uma replica em todos os pontos para analisar as variáveis na produção eletroquímica de cloro livre.

\section{OBJETIVO}

Este trabalho tem como objetivo empregar as técnicas estatísticas de planejamento de experimentos para quantificar o efeito das variáveis na produção eletroquímica de cloro livre.

\section{REVISÃO BIBLIOGRÁFICA}




\subsection{Utilização de cloro na degradação de poluentes orgânicos}

Comparando-se os métodos mais utilizados atualmente na desinfecção da água, os tratamentos com cloro e com hipoclorito são mais eficientes e de menor custo e apresentam a vantagem de possuírem ação residual, o que os difere dos tratamentos por irradiação ultravioleta e por ozônio (Kraft et al., 1999). A remoção de poluentes por métodos eletroquímicos tem recebido um interesse considerável nos últimos anos (Florêncio e Malpass, 2012). O Cloro empregado na desinfecção tem sido utilizado comercialmente de três formas: cloro líquido ou gasoso, hipoclorito de sódio e hipoclorito de cálcio. O cloro gasoso hidrolisa rapidamente em água para formas o ácido hipocloroso, conforme a Equação 1.

$$
\mathrm{Cl}_{2(g)}+\mathrm{H}_{2} \mathrm{O} \rightarrow \mathrm{HOCl}+\mathrm{H}^{+}+\mathrm{Cl}^{-}
$$

$\mathrm{O}$ ácido hipocloroso $(\mathrm{HOCl})$ é um ácido fraco tal que as concentrações relativas de ácido e a sua base conjugada são fortemente dependentes do pH (Equação 2). A dissociação de pH 6,5 a 8,5 é incompleta e as duas espécies, $\mathrm{HOCl}$ e $\mathrm{OCl}$-, estão presentes em pequena concentração (White, 1992). Abaixo de pH 6,5 não ocorre dissociação de HOCl. Acima de pH 8,5 todo ácido se dissocia ao íon hipocloroso. Considerando que o efeito germicida de $\mathrm{HOCl}$ é maior do que o de OCl-, preferem-se baixos pH para a desinfecção com esse agente.

$$
\mathrm{HOCl} \leftrightarrow \mathrm{OCl}^{-}+H^{+}\left(p K_{a}=7,5 a 25^{\circ} \mathrm{C}\right)
$$

Em muitos casos, para a degradação eletroquímica ocorrer em uma taxa significativa é necessário adicionar $\mathrm{NaCl}$ à mistura da reação, tendo duplo objetivo de: aumentar a condutividade do efluente (eletrólito de suporte) e a produção de $\mathrm{Cl}_{2}$ no ânodo. $\mathrm{O}$ que pode, posteriormente, formar espécies de cloro livre, as quais atuam na remoção de carga orgânica (Malpass et al.,2011). No entanto, o uso de $\mathrm{NaCl}$ pode ser problemático com reações subsequentes que podem resultar na formação de subprodutos do Cloro, que podem ser tóxicos e/ou carcinogênicos. A presença de tais compostos podem significativamente aumentar a toxicidade de efluentes tratados. Em estudos anteriores verificou-se que o uso de sais de cloreto de sódio como eletrólito suporte no processo e o emprego simultânea de UV diminuem a formação de organoclorados e reduz, portanto, a toxicidade dos produtos finais observados (Malpass et al., 2011).

\subsection{Planejamento de experimentos}

Segundo Costa e Almeida (2011), a essência de um bom planejamento consiste em projetar um experimento de forma que ele seja capaz de fornecer exatamente o tipo de informação que procuramos. Tendo selecionado os fatores importantes, o próximo passo seria avaliar quantitativamente sua influência sobre a resposta de interesse, bem como as possíveis interações de uns fatores com os outros. Para fazer isso com o mínimo de experimentos, podem-se empregar planejamentos fatoriais completos. Sua utilização tem como vantagem, reduz o número de experiências ou repetições e melhorar a qualidade da informação obtida através dos resultados, também, os fatores são analisados simultaneamente, assim, pode-se verificar e quantificar efeitos sinérgicos e antagônicos entre os fatores de interesse e é possível otimizar mais de uma resposta ao mesmo tempo. 
Quando o objetivo principal é otimizar um sistema, ou seja, maximizar ou minimizar algum tipo de resposta uma técnica conveniente é a metodologia de superfícies de resposta (RSM).

\section{PROCEDIMENTO EXPERIMENTAL}

Os ensaios foram realizados em uma célula eletroquímica de bancada com fluxo contínuo por meio de uma bomba dosadora peristáltica (Marca: Milan equipamento científicos; Modelo 202). A célula é composta por dois eletrodos um de Ti (contra elétrodo) e um elétrodo de trabalho de $\mathrm{Ti} / \mathrm{Ru}_{0,3} \mathrm{Ti}_{0,7} \mathrm{O}_{2}$ (De Nora, Brasil) com área de aproximadamente $1,5 \mathrm{~cm}^{2}$. A corrente elétrica foi fornecida por uma fonte estabilizada (Minipa MPL-1303M). A célula foi feita com vários espaçadores de Viton e Teflon, conforme a Figura 1.

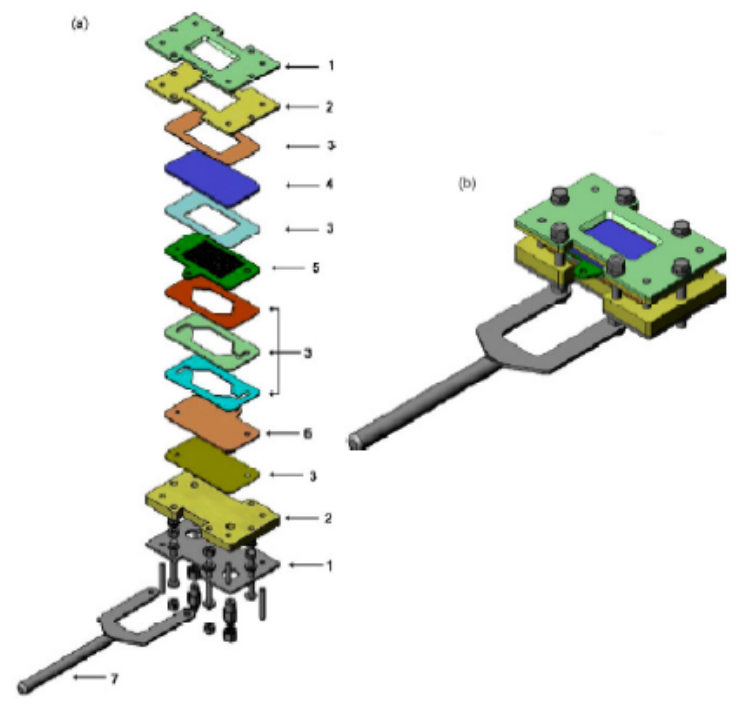

Figura 1 - Representação esquemática da célula eletroquímica em fluxo: (a) Visão explodida: (1) Chapa externa de aço; (2) Espaçadores de Teflon; (3) Espaçadores de Viton; (4) Janela de quartzo; (5) Rede de Ti (contra eletrodo); (6) Eletrodo de trabalho e (7) Suporte. (b) Célula montada.

Preparou-se inicialmente as soluções aquosas de cloreto de sódio $(\mathrm{NaCl})$ conforme especificadas as concentrações pelo planejamento experimental $2^{4}$. Em seguida, regulou-se o $\mathrm{pH}$ das soluções com auxilio de um phmetro. Posteriormente, ajustou-se o espaçamento da célula eletroquímica com auxilio de um paquímetro e, também, a corrente da fonte através de um amperímetro. $\mathrm{O}$ circuito foi ligado e depois de decorridos 30 minutos de reação retirou-se uma amostra para posterior quantificação de cloro livre produzido. Esta quantificação foi realizada por um método indireto através da titulação iodometria. Um volume de amostra de 1 $\mathrm{mL}$ foi retirado do processo após decorridos 30 minutos, e foi transferido para um erlenmeyer. Adicionou-se $5 \mathrm{~mL}$ de ácido acético e $10 \mathrm{~mL}$ de água destilada. Em seguida, adicionou-se cerca de $1 \mathrm{~g}$ de KI estimado com uma espátula, a solução apresentou uma cor amarelada, onde, longe da luz solar direta, titulou-se com a solução de $\mathrm{Na}_{2} \mathrm{~S}_{2} \mathrm{O}_{3}$ até a cor amarela do iodo quase ser descarregada. Posteriormente, adicionou-se o indicador de amido e titulou-se até que a cor azul esteja descarregada, ficando com uma tonalidade esbranquiçada. Anotou-se o volume gasto na titulação para posteriores cálculos, e assim, descobrir a produção de cloro livre pela célula eletroquímica. Vale salientar que a titulação foi realizada rapidamente, logo após a coleta da amostra e, também, sempre mantendo agitação. 
Um total de 32 experimentos foram realizados de acordo com um planejamento fatorial de $2^{4}$ (dois níveis e quatro variáveis) e com replica em todos os pontos. Os valores correspondentes aos níveis deste processo foram escolhidos utilizando o bom senso da equipe, como também, as limitações físicas dos equipamentos, os quais estão apresentados na Tabela 1. O delineamento experimental e análise de dados foram feitos usando o software STATISTICA.

Tabela 1 - Fatores variáveis com os valores codificados e reais.

\begin{tabular}{|c|c|c|c|}
\hline Variáveis & \multirow{2}{*}{ Símbolos } & \multicolumn{2}{|c|}{ Valores reais dos níveis codificados } \\
\cline { 3 - 4 } & & -1 & +1 \\
\hline Espaçamento (mm) & $\mathrm{X} 1$ & 3,90 & 7,35 \\
\hline Corrente (A) & $\mathrm{X} 2$ & 0,05 & 0,15 \\
\hline Concentração de NaCl (mol/L) & $\mathrm{X} 3$ & 0,1 & 1 \\
\hline $\mathbf{p H}$ & $\mathrm{X} 4$ & 3 & 11 \\
\hline
\end{tabular}

\section{RESULTADOS E DISCUSSÕES}

Para a análise das variáveis independentes e dependentes foi adotado um intervalo de confiança 95\%. O modelo simplificado obtido é expresso pela Equação 3.

$[C l]=967,12-38,8 X 1+311,3 X 2+65,4 X 3+29,9 X 1 X 3+25,5 X 2 X 3+29,9 X 2 X 4$

Para realização da análise das variáveis com efeitos mais significativos, plotou-se o gráfico de Pareto (Figura 2).

Figura 2 - Gráfico de Pareto.

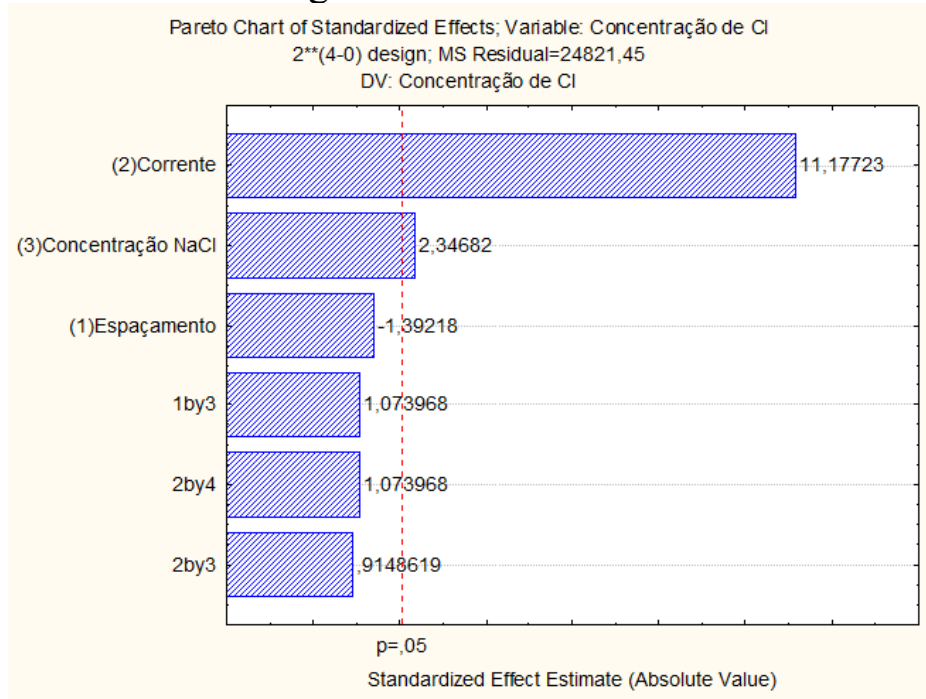

Vê-se que o efeito do coeficiente linear X2 (corrente) tem grande significância positiva no processo. Também, X3 (concentração de $\mathrm{NaCl}$ ) se mostra um pouco significativo. Já a variável X1 (espaçamento) não aparenta significância para o intervalo de confiança de 95\%, apresentando um comportamento negativo. Os termos eliminados e que se mostraram 


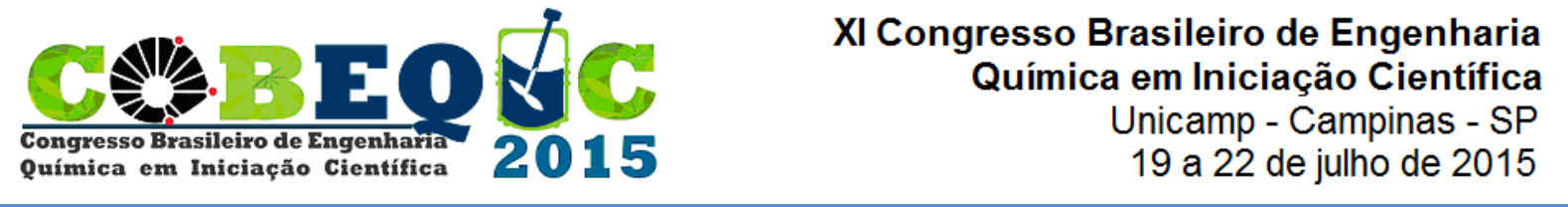

estatisticamente insignificantes foram as interações entre X1X2 (espaçamento e corrente), X1X4 (espaçamento e $\mathrm{pH}$ ) e X3X4 (concentração de $\mathrm{NaCl}$ e pH), e também a variável X4 $(\mathrm{pH})$. Plotou-se, também, o gráfico dos valores preditos versus os observados, para analisar o ajuste do modelo assim como, o gráfico dos resíduos (Figura 3).

Figura 3 - (a) Valores preditos versus observados. (b) Gráfico dos resíduos.
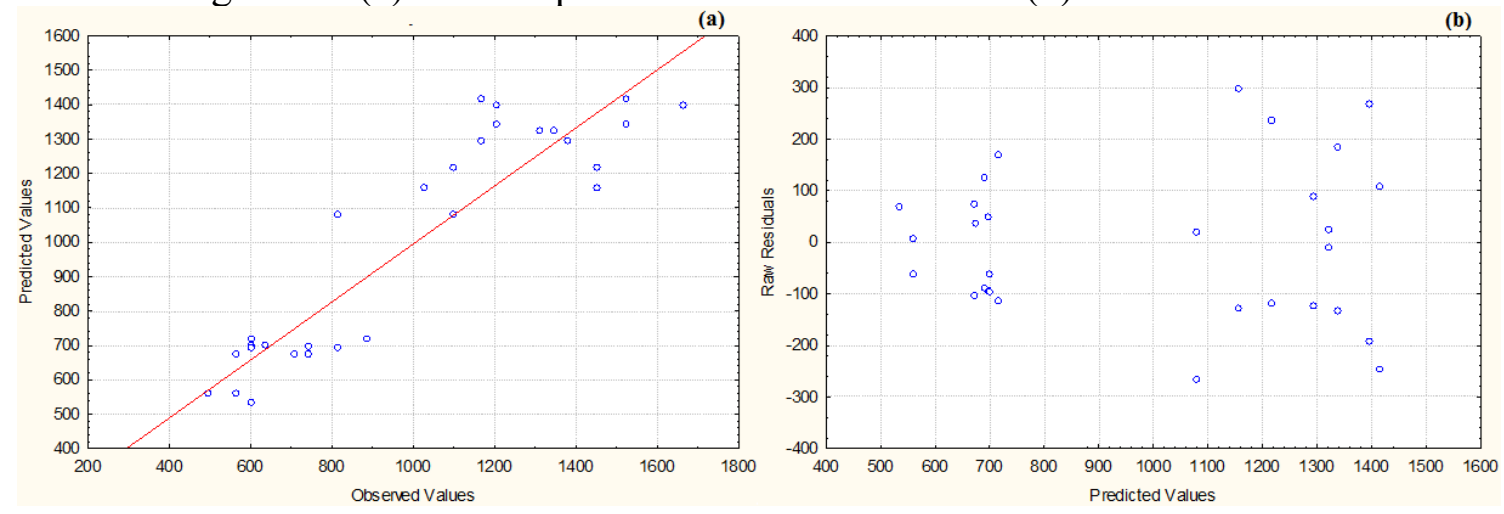

A partir dos gráficos verifica-se que o modelo é apropriado para análise dos dados e que as suposições são verdadeiras, pois os valores variam aleatoriamente em torno de zero, não observando nenhuma tendência. Em seguida plotou-se as superfícies de resposta das variáveis que se mostram mais significativas (Figuras 4).

Figura 4 - (a) Superfície de resposta entre corrente e concentração de $\mathrm{NaCl}$. (b) Superfície de resposta entre espaçamento e corrente.

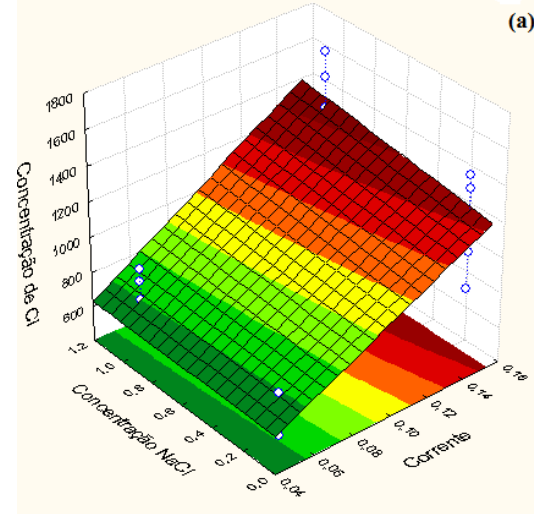

(a)
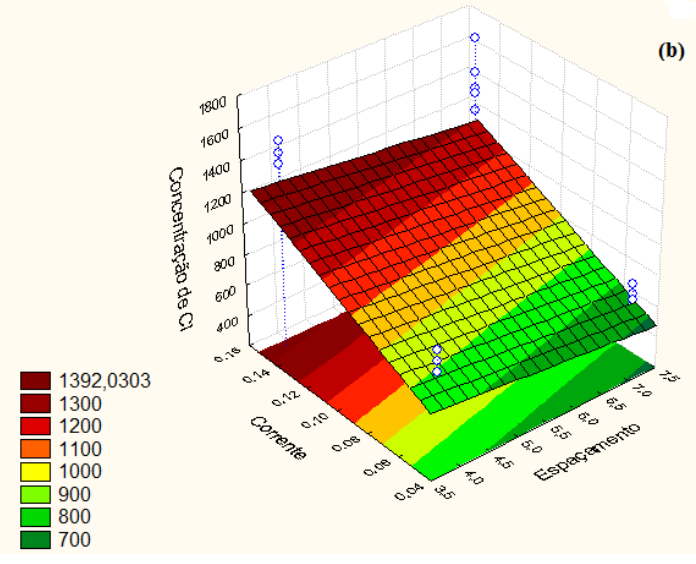

(b)

Pela analise da superfície de resposta é visto que quanto maior o valor da corrente maior o valor da resposta estudada, sendo que a melhor resposta é para o maior valor de concentração de $\mathrm{NaCl}$ e o menor valor de espaçamento.

\section{CONCLUSÃO}

O Design fatorial salienta que a variável mais significativa na produção de cloro residual é a corrente, a qual apresenta influência positiva. Já a segunda variável significante é a concentração de $\mathrm{NaCl}$, também com influencia positiva na resposta. As variáveis 


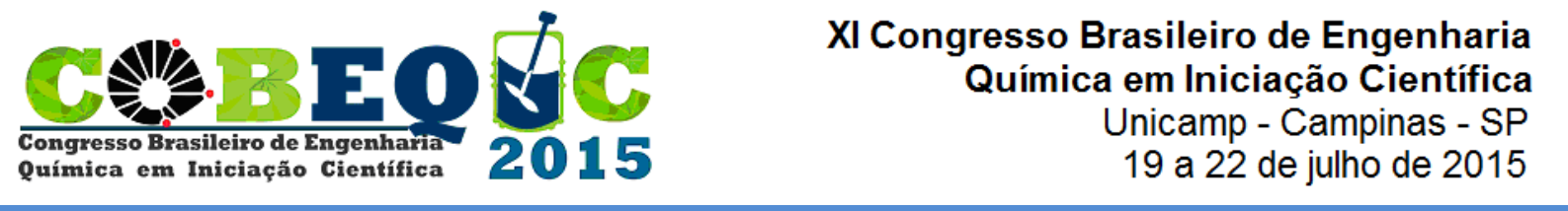

espaçamento e o pH não mostram muita influencia na reposta. Nenhuma interação entre as variáveis manifestaram significância na produção de cloro residual. Trabalhos a finalizar: Doravante, será necessário a execução do planejamento composto central (PCC) para verificação final das condições ótimas de produção por métodos eletroquímicos de cloro livre. Também será apresentada a degradação de poluentes orgânicos com as condições ótimas do processo para verificar a sua eficácia.

\section{REFERÊNCIAS}

BOX, G. E. P.; HUNTER, W. G. e HUNTER, J. S. Statistics for experimenters: na introduction to design, data analysis and model building. New York, Wiley, 1978.

COSTA, M. C. B. A; ALMEIDA, C. R. R. A importância do ensino da ferramenta de planejamento fatorial para estudantes de engenharia. In: Congresso Brasileiro de Educação em Engenharia, 39., 2011, Blumenau. Anais...Blumenau, 2011.

FLORÊNCIO, T.M.; MALPASS, G.R.P. A Brief Explanation about Environmental Licenses in Brazil. The Nexus. American Chemical Society. Sept/Oct. 2012.

KRAFT, A.; STADELMANN, M.; BLASCHKE, M.; KREYSIG, D.; SANDT, B.; SCHRODER, F.; RENNAU, J., 1999, Electrochemical water disinfection, Part I: Hypochlorite production from very dilute chloride solutions, Journal of Applied Electrochemistry, 29, 861-868.

MALPASS, G.R.P.; MIWA, D.W.; SANTOS, R.L.; VIEIRA, E.M.; MOTHEO, A.J. Unexpected toxicity decrease during photoelectrochemical degradation of atrazine with $\mathrm{NaCl}$. Environmental Chemistry Letters, v. 1, p. 1, 2011.

WHITE, G.C. (1992). Handbook of chlorination and alternative disinfctants. Nova York, Van Nostrand Reinhold, v.3. 